\title{
P-gp Inhibition by XL019, a JAK2 Inhibitor, Increases Apoptosis of Vincristine-treated Resistant KBV20C Cells with Increased p21 and pH2AX Expression
}

\author{
JI HYUN CHEON*, JI YEONG KIM*, BYUNG-MU LEE, HYUNG SIK KIM and SUNGPIL YOON \\ School of Pharmacy, Sungkyunkwan University, Suwon, Republic of Korea
}

\begin{abstract}
The present study was designed to identify conditions that could increase the sensitivity of resistant cancer cells to antimitotic drugs. We investigated whether a Janus kinase 2 (JAK2) inhibitor used in clinical trials, XLO19, sensitizes antimitotic drug-resistant KBV2OC cells. XLO19 reduced cellular viability and increased apoptosis in vincristinetreated KBV20C cells, independently of the JAK/signal transducer and activator of transcription (STAT) pathway. Based on the ATP-binding cassette protein B1 [ABCB1, Pglycoprotein $(P-g p)]$ inhibitory assay, we demonstrated that XL019 functions as a P-gp inhibitor in drug-resistant KBV20C cells. Considering that another JAK2 inhibitor, CEP-33779, also inhibited P-gp and sensitized drug-resistant cancer cells in a previous study, we concluded that JAK2 inhibitors can be used as P-gp inhibitors in drug-resistant cancer cells. Fluorescence-activated cell sorting, western blot, and annexin $V$ analyses were used to further investigate the mechanism of action of XLO19 in vincristine-treated KBV20C cells. XLO19 induced early apoptosis of KBV20C cells in response to vincristine treatment via increased $G_{2}$ phase arrest. Moreover, $G_{2}$ phase arrest and apoptosis of cells co-treated with vincristine and XLO19 resulted from the up-regulation of phosphorylated retinoblastoma protein ( $p R b), p 21$, and the DNA-damage protein, phosphorylated H2A histone family, member $X(p H 2 A X)$. Additionally, the P-gp-inhibitory effect of
\end{abstract}

*These Authors contributed equally to this study.

Correspondence to: Sungpil Yoon, Ph.D., School of Pharmacy, Sungkyunkwan University, 2066 Seobu-ro, Jangan-gu, Suwon, Gyeonggi-do, 16419, Republic of Korea. Tel: +82 1055024893 , Fax: +82 312928800, e-mail: syoon88@gmail.com and Hyung Sik Kim, Ph.D., School of Pharmacy, Sungkyunkwan University, 2066 Seobu-ro, Jangan-gu, Suwon, Gyeonggi-do, 16419, Republic of Korea. Tel: +82 312907789, Fax: +82 312928800, e-mail: hkims@skku.edu

Key Words: JAK2, XL019, CEP-33779, P-gp, drug-resistance.
XL019 was less than that of CEP-33779, and a more than 2fold higher dose was required to sensitize vincristine-treated KBV20C cells. Furthermore, lower doses of XL019 were required to sensitize KBV2OC cells to a degree similar to that obtained with the established P-gp inhibitor verapamil, suggesting that XL019 has higher specificity than verapamil. Our results showed that JAK2 inhibitors inhibited P-gp action via a direct binding mechanism, which was similar to that of verapamil. These findings indicate that JAK2 inhibitors may be promising therapeutics for the treatment of cancer that is resistant to antimitotic drugs.

Antimitotic drugs, that target different binding sites on tubulin, are widely used to treat different types of cancer (1-3). As patients develop multidrug resistance (MDR) to antimitotic drugs $(4,5)$, identifying the mechanism(s) underlying cell sensitization to antimitotic drugs would be an important step in the development of more effective treatments by designing approaches to increase drug-induced apoptosis. ATP-binding cassette protein B1 (ABCB1), more commonly known as $\mathrm{P}$ glycoprotein (P-gp) overexpression is mainly involved in resistance to antimitotic drugs $(6,7)$. $\mathrm{P}$-gp pumps such drugs out of cancer cells, and its overexpression is mainly responsible for MDR (7-9). Therefore, it is important to identify drugs or mechanisms that lead to reduction of P-gp overexpression in MDR cancer.

Janus kinase 2 (JAK2) is a protein that contributes to drug-resistant cancer $(10,11)$; therefore, JAK2 inhibitors have been developed to increase the efficacy of anticancer drugs $(12,13)$. The JAK2 inhibitor, CEP-33779, displays drug-sensitizing effects in P-gp-overexpressing drug-resistant cancer cells via P-gp-inhibitory activity (14). Thus, identifying the mechanisms underlying the effects of JAK2 inhibitors is important to broaden their clinical application.

In this study, we investigated whether XL019, another JAK2 inhibitor $(15,16)$, can sensitize P-gp-overexpressing MDR cancer cells. We also tested whether XL019 has P-gpinhibitory activity in MDR cancer cells. 


\section{Materials and Methods}

Reagents and cell culture. Rhodamine 123 and verapamil were purchased from Sigma-Aldrich (St. Louis, MO, USA). Vincristine was purchased from Enzo Life Sciences (Farmingdale, NY, USA). XL019 and CEP-33779 were purchased from Selleckchem (Houston, TX, USA).

Antibodies against phosphorylated signal transducer and activator of transcription (pSTAT3), STAT3, p21, and cleaved poly ADP ribose polymerase (C-PARP) were obtained from Cell Signaling Technology (Danvers, MA, USA). Antibodies against JAK2, $\beta$ actin, cyclin-dependent kinase 4 (CDK4), CDK2, phosphorylated retinoblastoma protein $(\mathrm{pRb})$, cyclin $\mathrm{B} 1$, and cyclin $\mathrm{E} 1$ were obtained from Santa Cruz Biotechnology (Santa Cruz, CA, USA). Antibody against phosphorylated H2A histone family, member X $(\mathrm{pH} 2 \mathrm{AX})$ was obtained from Abcam (Cambridge, UK).

Human oral squamous carcinoma cell line $\mathrm{KB}$ and its multidrugresistant subline KBV20C were obtained from Dr. Yong Kee Kim (College of Pharmacy, Sookmyung Women's University, Seoul, Republic of Korea) and have been previously described $(17,18)$. All cell lines were cultured in RPMI-1640 containing 10\% fetal bovine serum, $100 \mathrm{U} / \mathrm{ml}$ penicillin, and $100 \mu \mathrm{g} / \mathrm{ml}$ streptomycin (WelGENE, Daegu, Republic of Korea).

Microscopic observation. Cells grown in 6-well plates were treated with XL019, CEP-33779, verapamil, or vincristine for $48 \mathrm{~h}$. The medium was then removed, and phosphate-buffered saline (PBS) was added to each dish. Cells were examined immediately in two independent experiments using an ECLIPSE Ts2 inverted microscope (Nikon, Tokyo, Japan) with a $4 \times$ or a $10 \times$ objective lens (Nikon's Microscopy U).

Rhodamine uptake tests. The tests used to assess the ability of a drug to inhibit P-gp were based on a previously described method (19-21). Briefly, cells grown in 6-well plates were treated with XL019, CEP-33779, or verapamil and incubated for $24 \mathrm{~h}$ at $37^{\circ} \mathrm{C}$. Cells were then incubated with $2 \mu \mathrm{g} / \mathrm{ml}$ rhodamine for $1.5 \mathrm{~h}$ at $37^{\circ} \mathrm{C}$. The medium was removed, and the cells were washed with PBS. The stained cells were analyzed in two independent experiments using a Guava EasyCyte Plus Flow Cytometer (Merck Millipore, Billerica, MA, USA).

Fluorescence-activated cell sorting (FACS) analysis. FACS analysis was performed as previously described $(22,23)$. Cells were grown in 60-mm diameter dishes and treated with XL019, CEP-33779, verapamil, or vincristine for $24 \mathrm{~h}$. The cells were then dislodged by trypsin and pelleted by centrifugation. The pelleted cells were washed thoroughly with PBS, suspended in $75 \%$ ethanol for at least $1 \mathrm{~h}$ at $4{ }^{\circ} \mathrm{C}$, washed with PBS, and re-suspended in a cold propidium iodide (PI) staining solution ( $100 \mu \mathrm{g} / \mathrm{ml}$ RNase A and $50 \mu \mathrm{g} / \mathrm{ml}$ PI in PBS) for 30 $\min$ at $37^{\circ} \mathrm{C}$. The stained cells were analyzed in two independent experiments for relative DNA content using a Guava EasyCyte Plus Flow Cytometer (Merck Millipore, Billerica, MA, USA).

Annexin $V$ analysis. Annexin $\mathrm{V}$ analysis was conducted by using the annexin V-fluorescein isothiocyanate (FITC) staining kit (BD Bioscience, San Jose, CA, USA) as previously described (20, 23). Cells were grown in $60-\mathrm{mm}$ diameter dishes and treated with XL019, CEP-33779, verapamil, or vincristine for $24 \mathrm{~h}$. The cells were then dislodged by trypsin and pelleted by centrifugation. The pelleted cells were washed with PBS. Cells in $100 \mu \mathrm{l}$ of binding buffer received $5 \mu \mathrm{l}$ of annexin V-FITC and $5 \mu \mathrm{l}$ of PI and were then incubated for $15 \mathrm{~min}$ at room temperature. The stained cells were analyzed in two independent experiments using a Guava EasyCyte Plus Flow Cytometer (Merck Millipore).

Western blot analysis. Total cellular proteins were extracted as previously described $(23,24)$. Briefly, cells were grown in $60-\mathrm{mm}$ diameter dishes and treated with XL019, CEP-33779, verapamil, or vincristine for $24 \mathrm{~h}$. They were then washed twice with cold PBS and detached with scrapers. For total protein isolation, cells were suspended in PRO-PREP ${ }^{\mathrm{TM}}$ protein extract solution (iNtRON, Seongnam, Republic of Korea) and placed on ice for $30 \mathrm{~min}$. The suspension was collected after centrifugation at $15,000 \times \mathrm{g}$ for $5 \mathrm{~min}$ at $4^{\circ} \mathrm{C}$. Protein concentrations were measured by using a protein assay kit (Bio-Rad, Hercules, CA, USA) according to the manufacturer's instructions. The proteins were resolved by sodium dodecyl sulfate-polyacrylamide gel electrophoresis (SDS-PAGE) and subjected to western blot analysis as previously described $(23,24)$.

Cell viability assay. Cell proliferation was measured by a colorimetric assay using the EZ-CyTox cell viability assay kit (Daeillab, Seoul, Republic of Korea) according to the manufacturer's instructions. Briefly, cells grown in wells of 96-well plates were incubated with $10 \mu \mathrm{l}$ of EZ-CyTox solution for $1-2 \mathrm{~h}$ at $37^{\circ} \mathrm{C}$. Absorbance at $450 \mathrm{~nm}$ was determined immediately using the VERSA MAX Microplate Reader (Molecular Devices Corp., Sunnyvale, CA, USA). All experiments were performed at least in triplicate and repeated twice.

Wound-healing assay. KBV20C cells were plated on 60-mm diameter dishes and grown to $80-90 \%$ confluence. A scratch was made in the middle of the culture to enable detection of cellular growth. The cells were then treated for $24 \mathrm{~h}$ and $48 \mathrm{~h}$ with vincristine, verapamil or XL019, alone or in combination and examined under microscopy.

Statistical analysis. Data are presented as mean \pm standard deviation (S.D.). Statistical analysis was performed using Student's $t$-test and one-way analysis of variance (ANOVA) followed by a multiplecomparison test. Results were considered statistically significant compared to those of the control when $p<0.05$.

\section{Results}

Co-treatment with XL019 sensitizes drug-resistant KBV20C cancer cells to vincristine treatment. We tested whether the JAK2 inhibitor, XL019, can sensitize drug-resistant KBV20C cancer cells. As shown in Figure 1A, co-treatment with XL019 reduced the proliferation of vincristine-treated KBV20C cells when compared to single treatment using either vincristine or XL019. Moreover, reduced proliferation resulted in increased apoptosis (Figure 1B), as determined by increased C-PARP production in vincristine-XL019 cotreated cells. Considering that the JAK2 inhibitor CEP-33779 sensitizes drug-resistant KBV20C cancer cells to vincristine (14), we conclude that JAK2 inhibitors generally increase the sensitization of drug-resistant cancer cells to vincristine. 
A

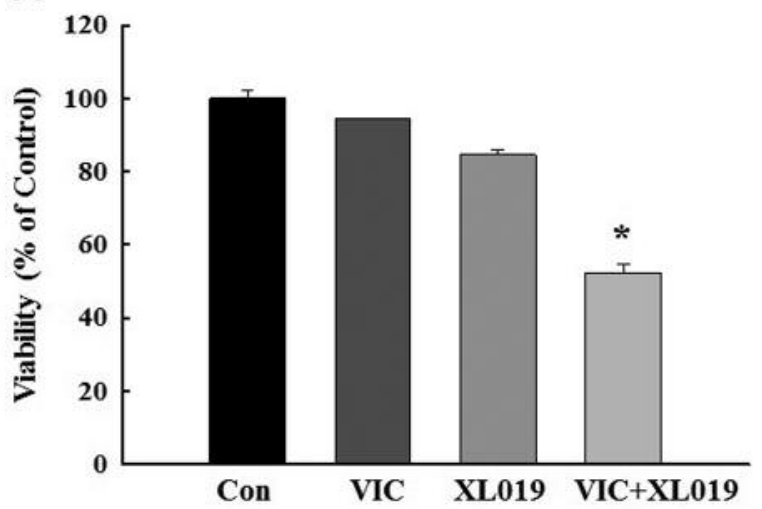

B

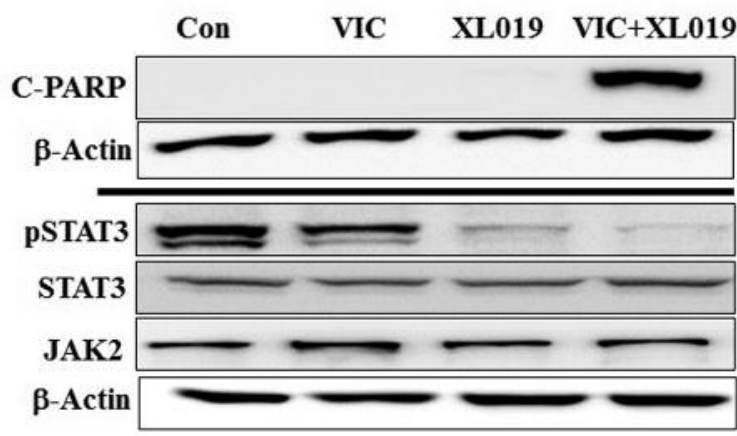

C
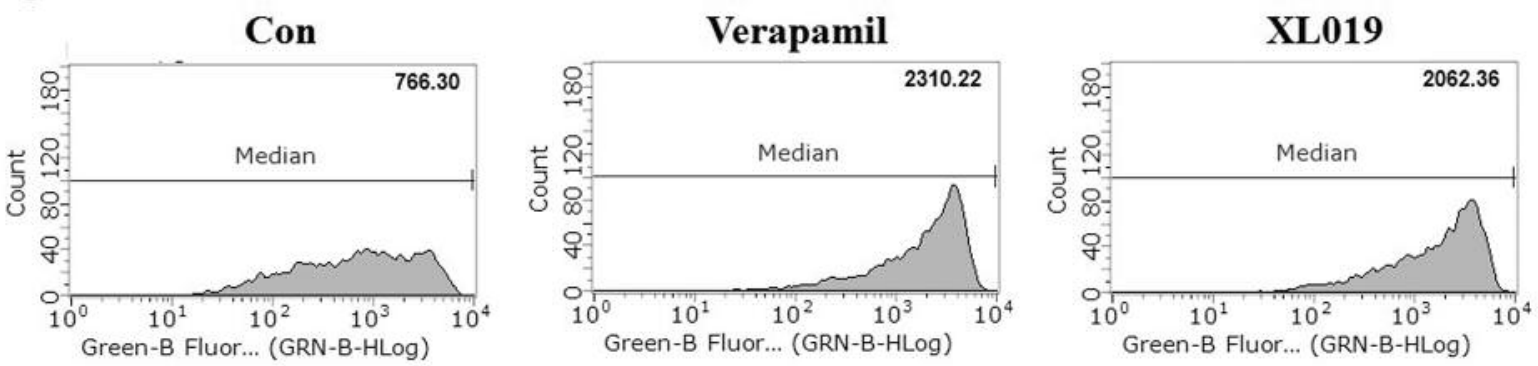

Figure 1. Co-treatment with the Janus kinase 2 (JAK2) inhibitor, XL019, sensitizes vincristine (VIC)-treated resistant KBV20C cancer cells through its P-glycoprotein (P-gp)-inhibitory activity. A: KBV20C cells were plated on 96-well plates and grown to $20-30 \%$ confluence. The cells were then stimulated for $72 \mathrm{~h}$ with $5 \mathrm{nM}$ vincristine, $5 \mu \mathrm{M}$ XL019, $5 \mathrm{nM}$ vincristine with $5 \mu \mathrm{M}$ XL019, or dimethylsulfoxide (DMSO; Con). A cell viability assay was performed as described in the Materials and Methods. B: KBV20C cells were plated on 60-mm diameter dishes and treated as described in (A) for $24 \mathrm{~h}$, western blot analysis was performed using antibodies against cleaved poly ADP ribose polymerase (C-PARP), phosphorylated signal transducer and activator of transcription (pSTAT3), STAT3, JAK2, and $\beta$-Actin. C: KBV20C cells were grown on 60 mm-diameter dishes and treated with $20 \mu \mathrm{M}$ verapamil, $5 \mu \mathrm{M}$ XL019, or 0.1\% DMSO (Con). After $24 \mathrm{~h}$, all cells were stained with rhodamine 123 and examined using fluorescenceactivated cell sorting (FACS) analysis, as described in the Materials and Methods. The data represent the mean \pm S.D. of at least two experiments repeated in triplicate experiments. Statistical analysis was conducted using one-way analysis of variance followed by multiple-comparison test: significantly different at *p<0.05 compared to the corresponding control.

JAK2 inhibitors are known to reduce STAT3 activation (25); therefore, we tested whether co-treatment with XL019 can reduce STAT3 activation in vincristine-treated KBV20C cells. As observed from Figure 1B, STAT3 activation significantly decreased after treatment with XL019 alone. However, cotreatment with vincristine did not reduce STAT3 activation further. The STAT3 and JAK2 total protein levels did not decrease in cells after co-treatment (Figure 1B), suggesting that co-treatment of vincristine-treated KBV20C cells with XL019 increased sensitization to vincristine, independently of the activation of the JAK/STAT signaling pathway.

The JAK2 inhibitor XL019 presents high P-gp-inhibiting activity. As CEP-33779 demonstrated P-gp-inhibitory activity by increasing the sensitization of vincristine-treated KBV20C cells $(12,14)$, we tested the P-gp-inhibitory activity of XL-019 in P-gp-overexpressing KBV20C cells. We evaluated whether XL019 increased the inhibition of Pgp substrate efflux. Rhodamine 123, a well-known P-gp substrate, was used to measure inhibition of P-gp (19-21). In this experiment, cellular accumulation of green fluorescence was indicative of intracellular accumulation of rhodamine 123. As shown in Figure 1C, XL019 showed high P-gpinhibitory activity, suggesting that XL019 functions as a Pgp inhibitor. The known P-gp inhibitor verapamil was used as a positive control (19-21). Upon comparison, a four-fold lower dose of XL019 was sufficient to induce inhibition of P-group comparable to that of verapamil. This suggests that the JAK2 inhibitor XL019 may bind to P-gp with higher specificity than does verapamil. This may also indicate that toxicity due to the use of P-gp inhibitor might be reduced using JAK2 inhibitor XL019. 
A

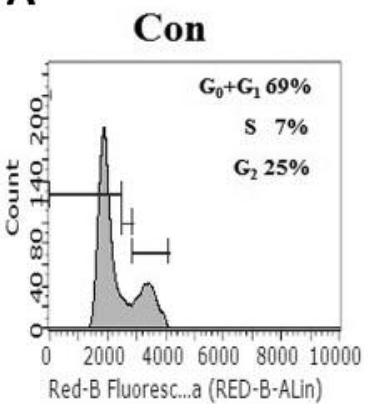

VIC

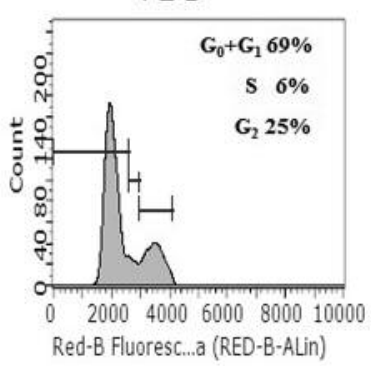

XL019

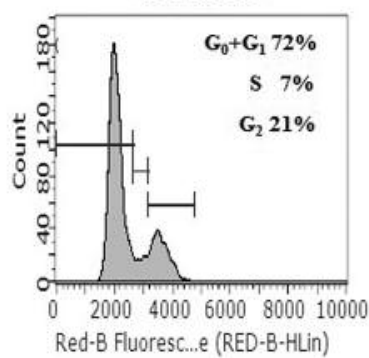

VIC+XL019

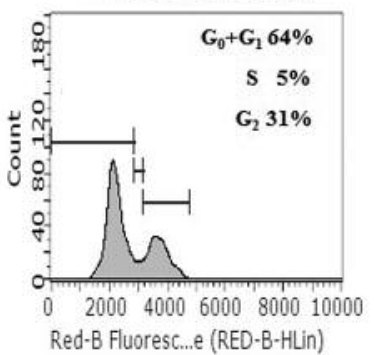

B
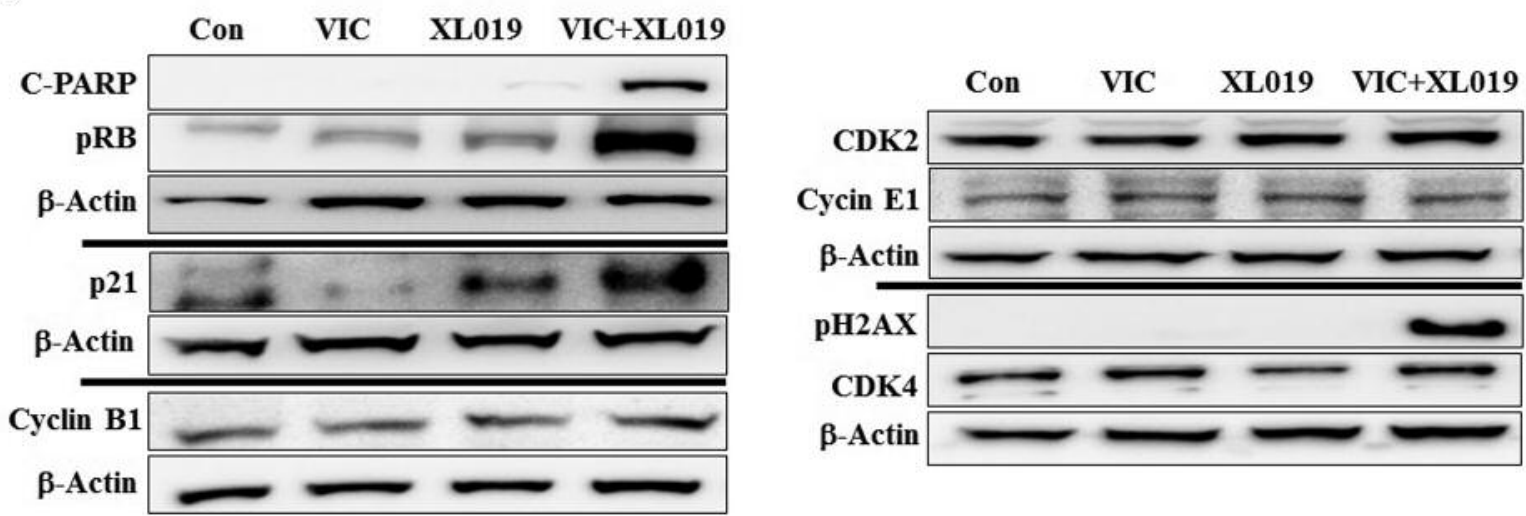

Figure 2. Co-treatment with XL019 sensitizes vincristine (VIC)-treated resistant KBV20C cancer cells through DNA damage and $G_{2}$ phase arrest. A: KBV20C cells were grown on 6-well plates and treated with $5 \mathrm{nM}$ vincristine, $5 \mu \mathrm{M} X \mathrm{~L} 019,5 \mathrm{nM}$ vincristine with $5 \mu \mathrm{M}$ XL019, or dimethylsulfoxide (Con) as described in (A) for $24 \mathrm{~h}$.fluorescence-activated cell sorting analyses were then performed as described in the Materials and Methods. B: KBV20C cells were plated on 60-mm diameter dishes and treated as described in (A). After 24 h, western blot analysis was performed using antibodies against cleaved poly ADP ribose polymerase (C-PARP), phosphorylated retinoblastoma protein ( $p R b)$, p21, cyclin B1, cyclin-dependent kinase 2 (CDK2), cyclin E1, phosphorylated H2A histone family, member X (pH2AX), CDK4, and $\beta$-Actin.

Altogether, our findings indicate that co-treatment with XL019 sensitized KBV20C cells to vincristine treatment, with higher P-gp-inhibitory activity, independently of inhibition of pSTAT3. This suggests that P-gp inhibition by XL019 plays an important role in the sensitization of KBV20C cells to antimitotic drugs.

Co-treatment with vincristine and XL019 induces $G_{2}$-arrest and increases $p H 2 A X$ and $p 21$ expression in KBV2OC cells. In order to further clarify the mechanism of sensitization of KBV20C cells to vincristine-XL109 cotreatment, we performed FACS analyses of treated cells. As shown in Figure 2A, co-treatment with XL019 and vincristine increased the number of cells arrested in the $G_{2}$ phase compared to that observed with single treatments with either agent. In order to further investigate the expression of proteins involved in $\mathrm{G}_{2}$ arrest $(26,27)$, we performed western blot analysis. As shown in Figure 2B, the production of the apoptotic marker, C-PARP, greatly increased. There was no significant difference in expression of cyclin proteins. However, the expression of $\mathrm{pH} 2 \mathrm{AX}$, a marker of DNA damage, was greatly increased following co-treatment. Increased p21 and pRB levels were also detected in co-treated cells (Figure 2B), suggesting that cell-cycle arrest by these proteins is involved in apoptotic sensitization. We conclude that DNA-damage and cell-cycle arrest signals ultimately increased apoptosis of vincristine-XL019 co-treated KBV20C cells via $\mathrm{G}_{2}$ arrest.

Co-treatment with XL019 increases the sensitization of vincristine-treated KBV20C cells to treatment with verapamil. In the next phase of our investigation, we tested the efficacy of XL019 as a P-gp inhibitor to sensitize KBV20C cells to 
A

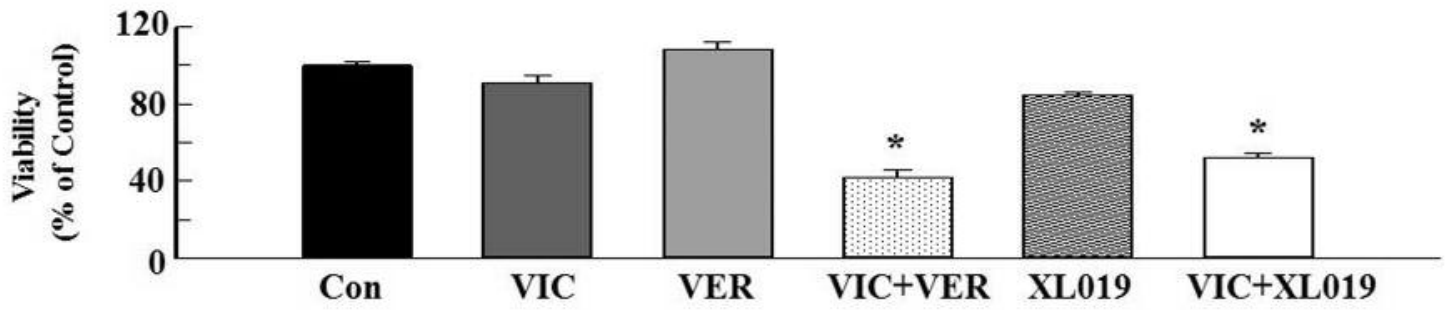

B
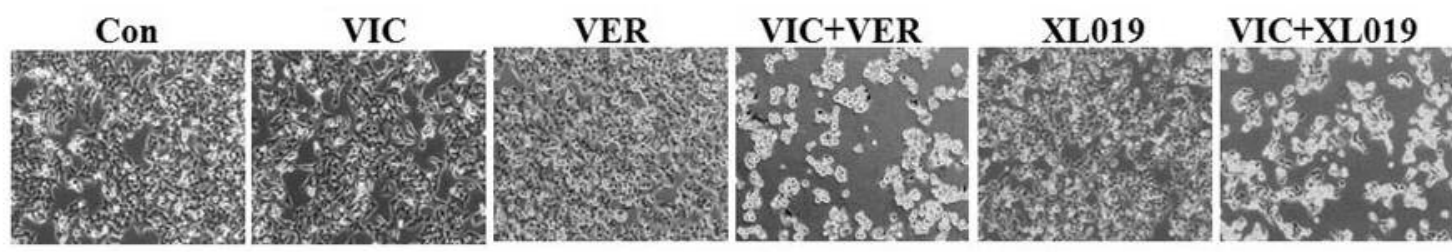

C
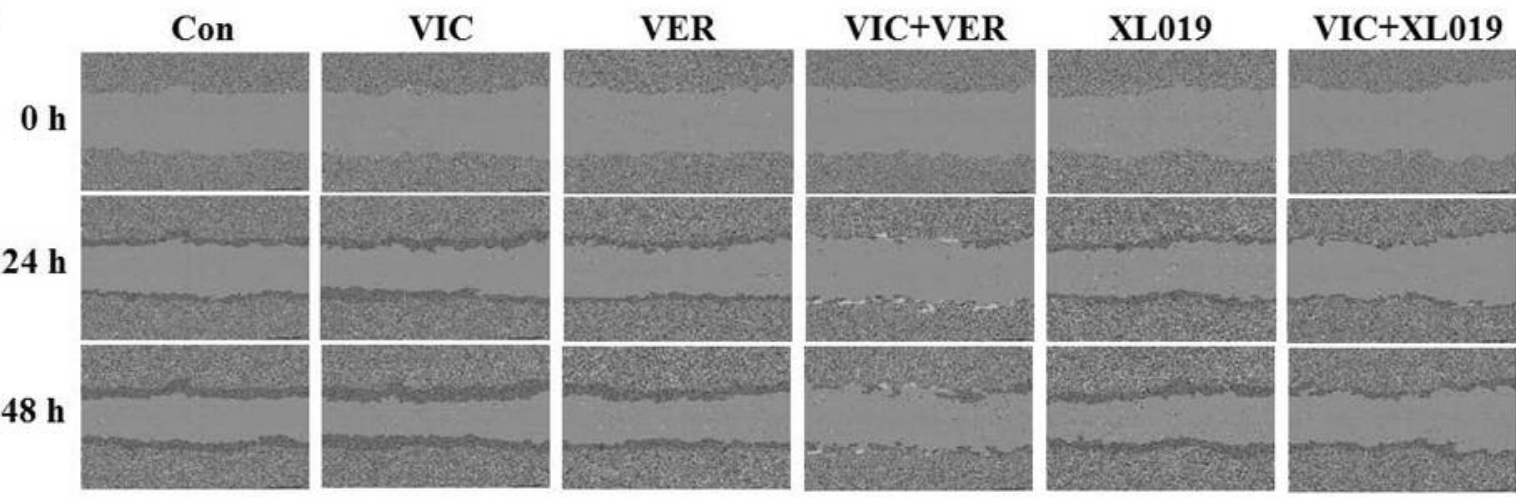

Figure 3. XL019 and verapamil have similar sensitizing efficacies in vincristine (VIC)-treated P-glycoprotein (P-gp)-overexpressing KBV20C cells. A: KBV20C cells were plated on 96-well plates and grown to 20-30\% confluence. The cells were then stimulated for $72 \mathrm{~h}$ with $5 \mathrm{nM}$ vincristine), $10 \mu M$ verapamil (VER), $5 \mathrm{nM}$ vincristine with $10 \mu \mathrm{M}$ verapamil, $5 \mu \mathrm{M}$ XL019, $5 \mathrm{nM}$ vincristine with $5 \mu \mathrm{M}$ XL019, or dimethylsulfoxide (Con). A cell viability assay was performed as described in Materials and Methods. B: KBV20C cells were grown on 6-well plates and treated as described in (A). After 2 days, all cells were observed using an inverted microscope at 100x magnification. C: KBV20C cells were plated on 60-mm diameter dishes and grown to 80-90\% confluence. A scratch was made in the middle of the culture to enable detection of cellular growth. The cells were treated for $24 \mathrm{~h}$ and $48 \mathrm{~h}$ as described in (A). They were subsequently observed using an inverted microscope. The data represent the mean $\pm S . D$. of at least two experiments repeated in triplicate experiments. Statistical analysis was conducted using one-way analysis of variance followed by multiple-comparison test: significantly different at $* p<0.05$ compared to the corresponding control.

vincristine-induced mitotic arrest. We compared the effects of XL019 with those of the well-known P-gp inhibitor, verapamil $(17,18)$. As shown in Figure 3A, $5 \mu \mathrm{M}$ XL019 or $10 \mu \mathrm{M}$ verapamil similarly sensitized the cells to vincristine, reducing KBV20C cell viability, and suggesting that a lower dose of XL019 is sufficient and more effective at sensitizing P-gpoverexpressing resistant cancer cells than the P-gp inhibitor verapamil. To confirm the sensitizing efficacy of XL019 and verapamil in vincristine-treated KBV20C cells, we performed microscopic observation of cells co-treated with vincristineXL019, and with verapamil-vincristine. As shown in Figure $3 \mathrm{~B}$, co-treatment of vincristine with $5 \mu \mathrm{M}$ XL019 or $10 \mu \mathrm{M}$ verapamil resulted in decreased numbers of KBV20C cells compared to those observed for single treatment with either of the inhibitors. Neither sensitive KB nor KBV20C cells responded to individual treatment with XL019 or verapamil alone, whereas the vincristine-sensitive parental KB cells did (data not shown), suggesting that XL019 has only P-gpinhibitory effects on $\mathrm{KB}$ and KBV20C cells. These data also confirmed that XL019 functions as a P-gp inhibitor similarly to verapamil.

Furthermore, the wound-healing assays indicated that wound healing barely occurred in vincristine-treated KBV20C cells co-treated with XL019 or verapamil, whereas wound healing in cells treated with either XL019 or verapamil alone was similar to that observed in control cells (Figure 3C). These results suggest that XL019 and verapamil have similar effects on cellular motility. These data indicate 
A

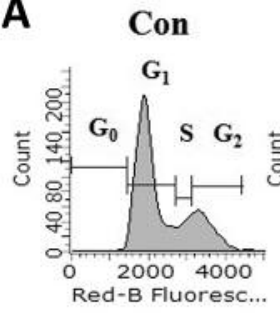

VIC

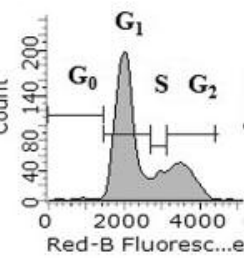

CEP-33779

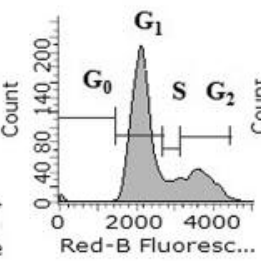

VIC+CEP

$\mathrm{G}_{1}$

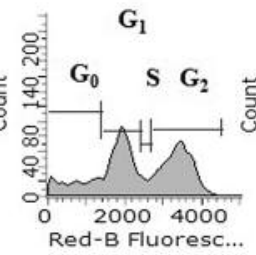

XL019

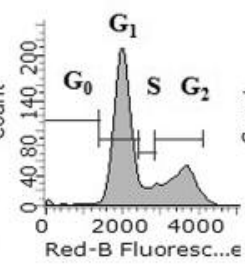

VIC+XL019

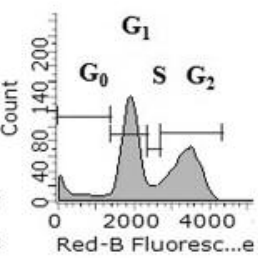

B
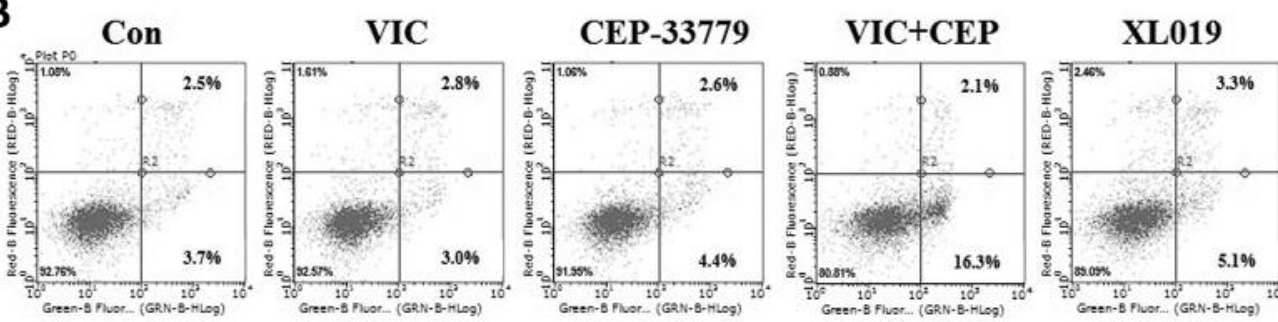

VIC+XL019

C

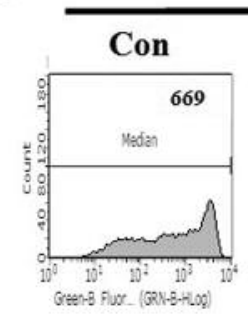

$24 \mathrm{~h}$

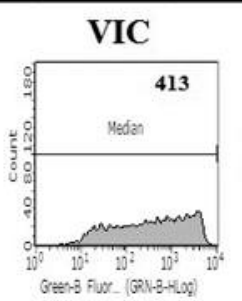

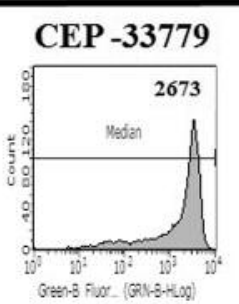

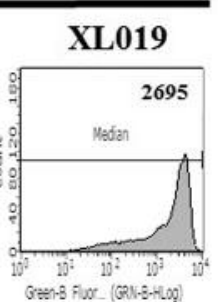

$3 \mathbf{h}$

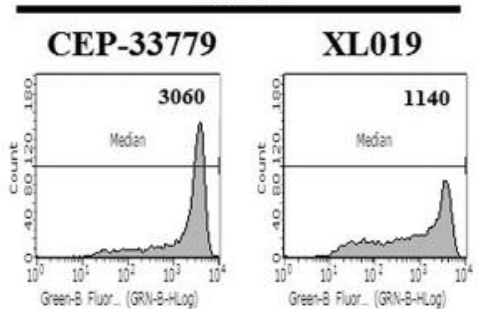

Figure 4. The Janus kinase 2 (JAK2) inhibitors CEP-33779 and XLO19 have different P-glycoprotein (P-gp)-inhibitory activities, reflecting their differing vincristine-sensitizing efficacy. A: KBV20C cells were grown on 6-well plates and treated with 5 nM vincristine (VIC), $2.5 \mu M$ CEP-33779, $5 \mathrm{nM}$ vincristine with $2.5 \mu \mathrm{M}$ CEP-33779 (VIC $+C E P$ ), $5 \mu \mathrm{M}$ XL019, $5 \mathrm{nM}$ vincristine with $5 \mu \mathrm{M}$ XL019, or dimethylsulfoxide (DMSO; Con). After $24 \mathrm{~h}$, fluorescence-activated cell sorting (FACS) analyses were performed as described in Materials and Methods. B: KBV20C cells were grown on 6-well plates and treated as in (A). After 24 h, annexin V analyses were performed as described in the Materials and Methods. C: KBV20C cells were grown on $60 \mathrm{~mm}$-diameter dishes and treated with $5 \mathrm{nM}$ vincristine, $5 \mu \mathrm{M}$ CEP-33779, $5 \mu \mathrm{M}$ XLO19, or 0.1\% DMSO (Con). After 3 or 24 h, all cells were stained with rhodamine123 and examined using FACS analysis as described in the Materials and Methods.

that co-treatment with the JAK2 inhibitor XL019 sensitizes KBV20C cells to vincristine treatment, with an efficacy similar to that of verapamil.

JAK2 inhibitors CEP-33779 and XLO19 have different P-gpinhibitory activities, reflecting their sensitizing efficacy. We showed that XL019 increased apoptosis of vincristine-treated KBV20C cells as determined by C-PARP production (Figures 1B and 2B). In more detailed quantitative analysis, we confirmed that co-treatment of cells with vincristine and XL019 increased the number of cells in the pre- $G_{1}$ phase and the number of cells in early phases of apoptosis compared with treatment with XL019 alone (Figure 4A and B). However, we detected no increase in the number of cells in a late stage of apoptosis in co-treated cells, suggesting that the sensitizing effects of XL019 mainly involve early apoptosis. Co-treatment with XL019 and vincristine increased early apoptosis of drug-resistant KBV20C cells via $\mathrm{G}_{2}$ cell-cycle arrest.

Finally, we compared the efficacy of two JAK2 inhibitors, XL019 and CEP-33779 (12, 14), in sensitizing P-gpoverexpressing KBV20C cells. Quantitative analysis of both FACS and annexin V (Figure 4A and B) showed that a lower dose of CEP-33779 induced $\mathrm{G}_{2}$ phase arrest and apoptosis of vincristine-treated KBV20C cells to a greater extent than that induced by XL019. These data suggest that CEP-33779 is more efficient in sensitizing cells to vincristine than XL019.

Next, we tested the P-gp-inhibitory activity of CEP-33779 and XL019 in P-gp-overexpressing KBV20C cells, since we assumed that the differene in the degree of $\mathrm{P}$-gp inhibition between XL019 and CEP-33779 is responsible for the difference in their sensitizing efficacy in vincristine-treated 
KBV20C cells. As shown in Figure 4C, CEP-33779 showed higher P-gp-inhibitory activity than XL019, while vincristine, used as a negative control, did not inhibit P-gp. In particular, when we reduced the treatment duration $(3 \mathrm{~h}$ vs. 24 h) with CEP-33779 and XL019, CEP-33779 more effectively inhibited P-gp activity in a shorter time than did XL019 (Figure 4C). These results suggest that JAK2 inhibitors exhibit different P-gp-inhibitory activity that depends on treatment duration. We assume that the efficacy of JAK2 inhibitors in the clinic may depend on both their dose and treatment duration.

Altogether, our findings indicated that XL019 presents Pgp-inhibitory functions that are independent of the JAK/STAT signaling pathway. Therefore, considering previous results with CEP-33779 (14), co-treatment of cells with JAK2 inhibitors can strongly prevent P-gp-mediated efflux of vincristine, thereby increasing the efficacy of antimitotic drugs in resistant cancer cells.

\section{Discussion}

JAK2 inhibitors are promising drugs for the treatment of triple-negative breast cancer (TNBC) and leukemia (10-13, $28,29)$. XL-019, a selective JAK2 inhibitor $(15,16)$, was recently tested in clinical trials $(15,16)$. Additionally, JAK2 inhibitors have been suggested for the treatment of chemotherapeutic-resistant TNBC $(28,29)$, as the JAK2 gene is highly amplified in this cancer type. In this study, we attempted to identify novel mechanisms of action of XL019 in sensitizing resistant cancer cells, as this may increase the application of XL019 for the treatment of various resistant cancer types.

The selective JAK2 inhibitor CEP-33779 exerts its P-gpinhibitory activity via direct binding and increases the sensitization of P-gp-overexpressing KBV20C cells to vincristine (14). Here, to our knowledge for the first time, we demonstrated that another JAK2 inhibitor, XL019, sensitizes vincristine-treated KBV20C cells through its P-gp-inhibitory activity. In detail, we demonstrated that a low dose of XL019 leads to inhibit pumping-out abilities of drugs via P-gp, as determined by rhodamine staining assay. Consistent with a previous study on the selective JAK2 inhibitor CEP-33779, which demonstrated its P-gp-inhibitory activity via direct binding (14), our results also suggest that XL019 directly binds to P-gp in drug-resistant KBV20C cancer cells, preventing P-gp-mediated drug efflux. Additionally, the effects of XL019 were independent of the JAK/STAT pathway in cells co-treated with vincristine and XL019, supporting our conclusion. We subsequently investigated the mechanism of action of JAK2 inhibitors on P-gp function in an in vitro model of drug resistance. Our data indicate that CEP-33779 had higher efficacy than XL019 at comparable low doses, implying that CEP33779 has a higher binding affinity. These results suggest that the increased efficacy of CEP-333779 in vincristine-treated KBV20C cells resulted from its increased P-gp-inhibitory activity. Considering that different JAK2 inhibitors can be used as alternative backup inhibitors for resistant cancer cells, our results show more solid evidence for applying JAK2 inhibitors to target P-gp in drug-resistant cancer cells. As most cancer types have been recognized as heterogeneous in various studies $(30,31)$, combination treatment seems to be important for dual-targeted therapy. Therefore, in a clinical setting, we hypothesize that both CEP33779 and XL019 may be applied to increase the drug sensitivity of both JAK2- and P-gp-overexpressing cancer cells in heterogeneous tumor cell populations.

In order to use XL019 as a P-gp inhibitor, its efficiency should be compared to that of the well-known P-gp inhibitor verapamil under vincristine-co-treatment. We found that $5 \mu \mathrm{M}$ XL019 had sensitizing effects to similar those induced by $10 \mu \mathrm{M}$ verapamil, suggesting that X1019 is more effective than verapamil in vincristine combination therapy. We, therefore, postulate that drug toxicity can be reduced by using XL019 against drug-resistant cancer. It is also possible that XL019 has other functions besides its P-gp-inhibiting activity when combined with vincristine, whereas verapamil has only P-gp-inhibitory functions. Further investigations are warranted to assess whether XL019 presents other functions in P-gp-overexpressing resistant cancer. Comparison among verapamil, CEP-33779, and XL019 showed that all three effectively sensitized KBV20C cells to vincristine. Considering that verapamil is a representative and wellknown P-gp inhibitor $(17,20,21)$, both JAK2 inhibitors should be regarded as P-gp inhibitors. Thus, we assume that in the clinic, both CEP-33779 and XL019 could be applied to sensitize not only JAK2-overexpressing cancer cells, but also P-gp-overexpressing cancer cells in heterogeneous cancer tissue populations such as TNBC. However, further in vivo studies using animal models are required to assess the efficacy of the vincristine-XL019 combined therapy before investigating its possible use in clinical trials.

In addition, XL019 in combination with vincristine induced cell apoptosis through $\mathrm{G}_{2}$ phase arrest. According to our results, p21 levels were highly increased in vincristineXL019 co-treated cells, indicating that cell-cycle arrest may play an important role in the effect of vincristine-XL019 cotreatment. Furthermore, the marker of DNA damage, $\mathrm{pH} 2 \mathrm{AX}$, cell-cycle protein $\mathrm{pRb}$, and the pro-apoptotic protein, C-PARP, increased after vincristine-XL019 cotreatment. This is indicative of a mechanism involving $\mathrm{G}_{2}$ phase arrest via DNA damage and an increase in the number of cells in the early phase of apoptosis.

Our results show that drug-resistant KBV20C cells that overexpress P-gp can be sensitized to antimitotic drug treatment with the JAK2 inhibitor XL019. As XL019 is already being used in clinical trials, the urgent need for 
drugs to treat antimitotic drug-resistant cancer can be efficiently addressed, and JAK2 inhibitors may be used to treat $\mathrm{P}$-gp-overexpressing resistant patients at a relatively faster pace.

\section{Conflicts of Interests}

The Authors declare no conflict of interests in regard to this study.

\section{Acknowledgements}

The Authors thank Yujin Park for help in technical support and preparation of the article. This research was supported by the National Research Foundation of Korea (NRF) funded by the Ministry of Education (NRF-2017R1D1A1B03029158).

\section{References}

1 Jordan MA and Wilson L: Microtubules as a target for anticancer drugs. Nat Rev Cancer 4: 253-265, 2004.

2 Kim JH, Yoo HI, Kang HS, Ro J and Yoon S: Salinomycin sensitizes antimitotic drugs-treated cancer cells by increasing apoptosis via the prevention of G2 arrest. Biochem Biophys Res Commun 418: 98-103, 2012.

3 McGrogan BT, Gilmartin B, Carney DN and McCann A: Taxanes, microtubules and chemoresistant breast cancer. Biochim Biophys Acta 1785: 96-132, 2008.

4 Araque Arroyo P, Ubago Perez R, Cancela Diez B, Fernandez Feijoo MA, Hernandez Magdalena J and Calleja Hernandez MA: Controversies in the management of adjuvant breast cancer with taxanes: review of the current literature. Cancer Treat Rev 37: 105-110, 2011.

5 Matsuo K, Bond VK, Im DD and Rosenshein NB: Prediction of chemotherapy response with platinum and taxane in the advanced stage of ovarian and uterine carcinosarcoma: a clinical implication of in vitro drug resistance assay. Am J Clin Oncol 33: 358-363, 2010.

6 Chen Z, Shi T, Zhang L, Zhu P, Deng M, Huang C, Hu T, Jiang $\mathrm{L}$ and Li J: Mammalian drug efflux transporters of the ATP binding cassette $(\mathrm{ABC})$ family in multidrug resistance: A review of the past decade. Cancer Lett 370: 153-164, 2016.

7 Xia CQ and Smith PG: Drug efflux transporters and multidrug resistance in acute leukemia: therapeutic impact and novel approaches to mediation. Mol Pharmacol 82: 1008-1021, 2012.

8 Shukla S, Wu CP and Ambudkar SV: Development of inhibitors of ATP-binding cassette drug transporters: present status and challenges. Expert Opin Drug Metab Toxicol 4: 205-223, 2008.

9 Yang K, Wu J and Li X: Recent advances in the research of Pglycoprotein inhibitors. Biosci Trends 2: 137-146, 2008.

10 Buchert M, Burns CJ and Ernst M: Targeting JAK kinase in solid tumors: emerging opportunities and challenges. Oncogene 35: 939-951, 2016.

$11 \mathrm{Xu} \mathrm{Y,} \mathrm{Zhang} \mathrm{J,} \mathrm{Wu} \mathrm{J,} \mathrm{Zhong} \mathrm{S} \mathrm{and} \mathrm{Li} \mathrm{H:} \mathrm{Inhibition} \mathrm{of} \mathrm{JAK2}$ reverses paclitaxel resistance in human ovarian cancer cells. Int J Gynecol Cancer 25: 1557-1564, 2015.

12 Dugan BJ, Gingrich DE, Mesaros EF, Milkiewicz KL, Curry MA, Zulli AL, Dobrzanski P, Serdikoff C, Jan M, Angeles TS, Albom MS, Mason JL, Aimone LD, Meyer SL, Huang Z, Wells-
Knecht KJ, Ator MA, Ruggeri BA and Dorsey BD: A selective, orally bioavailable 1,2,4-triazolo[1,5-a]pyridine-based inhibitor of Janus kinase 2 for use in anticancer therapy: discovery of CEP-33779. J Med Chem 55: 5243-5254, 2012.

13 Ringel F, Kaeda J, Schwarz M, Oberender C, Grille P, Dörken B, Marque F, Manley PW, Radimerski T and le Coutre P: Effects of JAK2 type 1 inhibitors NVP-BSK805 and NVP-BVB808 on $J A K 2$ mutation-positive and $B C R-A B L$-positive cell lines. Acta Haematol 132: 75-86, 2014.

14 Tang SJ, Chen LK, Wang F, Zhang YK, Huang ZC, To KK, Wang XK, Talele TT, Chen ZS, Chen WQ and Fu LW: CEP33779 antagonizes ATP-binding cassette subfamily B member 1mediated multidrug resistance by inhibiting its transport function. Biochem Pharmacol 91: 144-156, 2014.

15 Forsyth T, Kearney PC, Kim BG, Johnson HW, Aay N, Arcalas A, Brown DS, Chan V, Chen J, Du H, Epshteyn S, Galan AA, Huynh TP, Ibrahim MA, Kane B, Koltun ES, Mann G, Meyr LE, Lee MS, Lewis GL, Noguchi RT, Pack M, Ridgway BH, Shi X, Takeuchi CS, Zu P, Leahy JW, Nuss JM, Aoyama R, Engst S, Gendreau SB, Kassees R, Li J, Lin SH, Martini JF, Stout T, Tong P, Woolfrey J, Zhang $\mathrm{W}$ and Yu P: SAR and in vivo evaluation of 4-aryl-2aminoalkylpyrimidines as potent and selective Janus kinase 2 (JAK2) inhibitors. Bioorg Med Chem Lett 22: 7653-7658, 2012.

16 Verstovsek S, Tam CS, Wadleigh M, Sokol L, Smith CC, Bui LA, Song C, Clary DO, Olszynski P, Cortes J, Kantarjian H and Shah NP: Phase I evaluation of XL019, an oral, potent, and selective JAK2 inhibitor. Leuk Res 38: 316-322, 2014.

17 Cheon JH, Lee BM, Kim HS and Yoon S: Highly halavenresistant KBV20C cancer cells can be sensitized by co-treatment with fluphenazine. Anticancer Res 36: 5867-5874, 2016.

18 Park Y, Son JY, Lee BM, Kim HS and Yoon S: Highly eribulinresistant KBV20C oral cancer cells can be sensitized by cotreatment with the third-generation P-glycoprotein inhibitor, elacridar, at a low dose. anticancer res 37: 4139-4146, 2017.

19 Choi AR, Kim JH, Cheon JH, Kim HS and Yoon S: Attenuation of colchicine toxicity in drug-resistant cancer cells by co-treatment with anti-malarial drugs. Anticancer Res 36: 5859-5866, 2016.

20 Choi AR, Kim JH, Woo YH, Kim HS and Yoon S: Anti-malarial drugs primaquine and chloroquine have different sensitization effects with anti-mitotic drugs in resistant cancer cells. Anticancer Res 36: 1641-1648, 2016.

21 Lim JS, Park Y, Lee BM, Kim HS and Yoon S: Co-treatment with celecoxib or ns398 strongly sensitizes resistant cancer cells to antimitotic drugs independent of P-gp Inhibition. Anticancer Res 36: 5063-5070, 2016.

22 Choi AR, Kim JH, Woo YH, Cheon JH, Kim HS and Yoon S: Co-treatment of LY294002 or MK-2206 with AZD5363 attenuates AZD5363-induced increase in the level of phosphorylated AKT. Anticancer Res 36: 5849-5858, 2016.

23 Park EY, Woo Y, Kim SJ, Kim DH, Lee EK, De U, Kim KS, Lee J, Jung JH, Ha KT, Choi WS, Kim IS, Lee BM, Yoon S, Moon HR and Kim HS: Anticancer effects of a new SIRT Inhibitor, MHY2256, against human breast cancer MCF-7 cells via regulation of MDM2-p53 Binding. Int J Biol Sci 12: 1555-1567, 2016.

24 Kim JH, Choi AR, Kim YK, Kim HS and Yoon S: Low amount of salinomycin greatly increases AKT activation, but reduces activated p70S6K levels. Int J Mol Sci 14: 17304-17318, 2013.

25 Banerjee $\mathrm{K}$ and Resat H: Constitutive activation of STAT3 in breast cancer cells: A review. Int J Cancer 138: 2570-2578, 2016. 
26 Choi AR, Jo MJ, Jung MJ, Kim HS and Yoon S: Selenate specifically sensitizes drug-resistant cancer cells by increasing apoptosis via $\mathrm{G} 2$ phase cell-cycle arrest without P-GP inhibition. Eur J Pharmacol 764: 63-69, 2015.

27 Kim JH, Chae M, Choi AR, Sik Kim H and Yoon S: SP600125 overcomes antimitotic drug-resistance in cancer cells by increasing apoptosis with independence of P-gp inhibition. Eur J Pharmacol 723: 141-147, 2014.

28 Balko JM, Schwarz LJ, Luo N, Estrada MV, Giltnane JM, Dávila-González D, Wang K, Sánchez V, Dean PT, Combs SE, Hicks D, Pinto JA, Landis MD, Doimi FD, Yelensky R, Miller VA, Stephens PJ, Rimm DL, Gómez H, Chang JC, Sanders ME, Cook RS and Arteaga CL: Triple-negative breast cancers with amplification of JAK2 at the 9p24 locus demonstrate JAK2specific dependence. Sci Transl Med 8: 334ra353, 2016.

29 Barrett MT, Anderson KS, Lenkiewicz E, Andreozzi M, Cunliffe HE, Klassen CL, Dueck AC, McCullough AE, Reddy SK, Ramanathan RK, Northfelt DW and Pockaj BA: Genomic amplification of 9p24.1 targeting JAK2, PD-L1, and PD-L2 is enriched in high-risk triple-negative breast cancer. Oncotarget 6 : 26483-26493, 2015.

30 de Bruin EC, McGranahan N, Mitter R, Salm M, Wedge DC, Yates L, Jamal-Hanjani M, Shafi S, Murugaesu N, Rowan AJ, Grönroos E, Muhammad MA, Horswell S, Gerlinger M, Varela I, Jones D, Marshall J, Voet T, Van Loo P, Rassl DM, Rintoul
RC, Janes SM, Lee SM, Forster M, Ahmad T, Lawrence D, Falzon M, Capitanio A, Harkins TT, Lee CC, Tom W, Teefe E, Chen SC, Begum S, Rabinowitz A, Phillimore B, Spencer-Dene B, Stamp G, Szallasi Z, Matthews N, Stewart A, Campbell P and Swanton C: Spatial and temporal diversity in genomic instability processes defines lung cancer evolution. Science 346: 251-256, 2014.

31 Gerlinger M, Rowan AJ, Horswell S, Math M, Larkin J, Endesfelder D, Gronroos E, Martinez P, Matthews N, Stewart A, Tarpey P, Varela I, Phillimore B, Begum S, McDonald NQ, Butler A, Jones D, Raine K, Latimer C, Santos CR, Nohadani M, Eklund AC, Spencer-Dene B, Clark G, Pickering L, Stamp G, Gore M, Szallasi Z, Downward J, Futreal PA and Swanton C: Intratumor heterogeneity and branched evolution revealed by multiregion sequencing. N Engl J Med 366: 883-892, 2012.

Received August 24, 2017

Revised September 21, 2017

Accepted September 25, 2017 